\title{
Diagnostic use of lung ultrasound compared to chest radiograph for suspected pneumonia in a resource-limited setting
}

\author{
Yogendra Amatya', Jordan Rupp ${ }^{2}$, Frances M. Russell ${ }^{3}$, Jason Saunders ${ }^{3}$, Brian Bales ${ }^{2}$ and Darlene R. House ${ }^{1,3^{*}}$
}

\begin{abstract}
Background: Lung ultrasound is an effective tool for diagnosing pneumonia in developed countries. Diagnostic accuracy in resource-limited countries where pneumonia is the leading cause of death is unknown. The objective of this study was to evaluate the sensitivity of bedside lung ultrasound compared to chest $\mathrm{X}$-ray for pneumonia in adults presenting for emergency care in a low-income country.

Methods: Patients presenting to the emergency department with suspected pneumonia were evaluated with bedside lung ultrasound, single posterioranterior chest radiograph, and computed tomography (CT). Using CT as the gold standard, the sensitivity of lung ultrasound was compared to chest X-ray for the diagnosis of pneumonia using McNemar's test for paired samples. Diagnostic characteristics for each test were calculated.

Results: Of 62 patients included in the study, 44 (71\%) were diagnosed with pneumonia by CT. Lung ultrasound demonstrated a sensitivity of $91 \%$ compared to chest $X$-ray which had a sensitivity of $73 \%(p=0.01)$. Specificity of lung ultrasound and chest $X$-ray were 61 and $50 \%$ respectively.
\end{abstract}

Conclusions: Bedside lung ultrasound demonstrated better sensitivity than chest $\mathrm{X}$-ray for the diagnosis of pneumonia in Nepal.

Trial registration: ClinicalTrials.gov, registration number NCT02949141. Registered 31 October 2016.

Keywords: Lung ultrasound, Pneumonia, Nepal, Diagnosis, Developing countries

\section{Background}

Pneumonia is one of the leading causes of death worldwide, and in low-income countries, it is the leading cause of death [1]. These deaths may be prevented by early detection and targeted antibiotic therapy [2]. However, the diagnosis of pneumonia is not always clear on presentation to health care facilities. Imaging usually includes a chest X-ray or, in some cases, a thoracic computed tomography $(\mathrm{CT})$ scan. While the latter has the highest sensitivity, it is associated with high costs and higher doses of radiation [3, 4]. Because of these limitations, chest X-ray continues to be the main diagnostic modality for pneumonia despite its low sensitivity (43-78\%) [4-8].

\footnotetext{
* Correspondence: dhouse@iu.edu

'Department of General Practice and Emergency Medicine, Patan Academy of Health Sciences, PO Box 26500, Kathmandu, Nepal

${ }^{3}$ Department of Emergency Medicine, Indiana University School of Medicine, Indianapolis, IN, USA

Full list of author information is available at the end of the article
}

Several studies in high-income countries and one study by Liu et al. in China, a middle-income country, have shown ultrasound to be a reliable tool to diagnose pneumonia with a higher sensitivity and specificity than chest X-ray [8-13]. Ultrasound is a safe, portable, and inexpensive diagnostic modality that is widely used in resource-limited countries [14]. In remote areas with limited access to X-rays, clinicians are reliant on their physical examination, which also has limited sensitivity (47-69\%), and therefore, ultrasound for diagnosing pneumonia has the potential to be very useful in this setting [15]. Even in urban settings, use of bedside ultrasound can alleviate diagnostic dilemmas with critically ill patients, especially where facilities lack portable X-rays. However, the diagnostic accuracy of ultrasound to diagnose pneumonia in low-income countries like Nepal has not yet been studied. Results from studies in highincome countries are difficult to extrapolate to settings 
like Nepal where there are not only higher rates of pneumonia, but also high rates of tuberculosis (245 per 100,000) and chronic obstructive pulmonary disease (estimated $23-43 \%$ prevalence), which may make clinical and radiographic diagnosis of pneumonia more difficult [16-20].

Therefore, the objective of this study was to evaluate the sensitivity of bedside lung ultrasound compared to chest X-ray for the diagnosis of pneumonia in patients presenting to the emergency department in Nepal.

\section{Methods}

\section{Study design}

A prospective, convenience sample of patients presenting to the emergency department with suspected pneumonia were enrolled when a trained sonographer was present. Patients were enrolled from November 2016 to March 2017. This study was approved by both Vanderbilt University Institutional Review Board and Nepal Health Research Council Ethical Review Board. The study was registered with ClinicalTrials.gov, registration number NCT02949141. Written informed consent in Nepali was obtained from patients prior to enrollment. Lung ultrasounds and chest CT scans were provided free of charge to the patient.

\section{Study setting and population}

Located in the Kathmandu Valley, Patan Hospital is a large urban hospital with a 35-bed emergency department. The emergency department has an annual volume of approximately 36,000 patients with a $20 \%$ admission rate.

We included patients age 18 or older with suspected pneumonia. Patients had at least three of the following signs or symptoms: temperature greater than $38^{\circ} \mathrm{C}$ or history of fever, cough, dyspnea, tachypnea (respiratory rate greater than 20), or oxygen saturation lower than $92 \%$.

\section{Study protocol}

Four emergency department faculty and resident physicians were trained on the use of lung ultrasound for diagnosing pneumonia. This training included a 1-h lecture on lung ultrasound followed by a week of performing lung ultrasounds in the department. Prior to enrollment, clinicians each performed five lung ultrasounds with interpretations. These pre-enrollment scans were reviewed by an expert sonographer with registered diagnostic medical sonographer certification to ensure adequate skill in acquiring and interpreting lung ultrasounds. Prior to enrollment, a kappa analysis revealed good inter-rater reliability between the trainees and the expert sonographer $(k=0.67)$.

Once consent was obtained, the clinician recorded patient demographics, symptoms, and physical examination findings on a standardized data collection sheet.
After clinical evaluation, a bedside lung ultrasound was performed. Sonographers were not blinded to clinical information. A Sonosite M Turbo (Fujifilm Sonosite, Inc.) ultrasound machine with a curvilinear probe was used. In accordance with previous literature, the ultrasound examination included ten views: two anterior views and two lateral views (one including the costophrenic angle), and one posterior view on each hemithorax $[10,21]$. The physician recorded ultrasound findings and interpretation. An ultrasound diagnosis of pneumonia was defined as the presence of unilateral $B$ lines or subpleural lung consolidation.

All patients had a single posterioranterior (PA) chest $\mathrm{X}$-ray as a part of the standard evaluation. Two-view PA and lateral chest X-rays are not routinely ordered in this setting as it requires patients to pay for a second X-ray. For this reason, ultrasound was compared to the single PA view only. Clinicians performing the lung ultrasound were blinded to the results of the chest X-ray. The chest $\mathrm{X}$-ray was read by a board-certified radiologist, who was blinded to the clinical presentation and the results of any other imaging. Patients then underwent chest CT, as the diagnostic standard to evaluate for pneumonia. The CT was also read by a radiologist who was blinded to the clinical presentation and results of the previous studies. If the radiology read was indeterminate, the radiologist was asked specifically regarding the presence of pneumonia and the response was recorded. Chest Xray and $\mathrm{CT}$ interpretations were collected for statistical analysis.

\section{Data analysis}

Sensitivity, specificity, positive and negative predictive values, and likelihood ratios for both lung ultrasound and chest X-ray were calculated. According to McNemar's test comparing proportions of paired samples, a sample size of 62 patients was needed to detect a $20 \%$ difference between chest X-ray and lung ultrasound based on a sensitivity of chest X-ray of $70 \%$. Using $\mathrm{R}$ statistical program (version 3.3.2), McNemar's test was used to evaluate any statistical difference in sensitivity between chest X-ray and lung ultrasound. A kappa analysis was done to determine inter-rater reliability for ultrasound interpretations between the clinician sonographer and an expert sonographer with registered diagnostic medical sonographer certification. The expert sonographer was blinded to the clinician's interpretation and results of other imaging.

\section{Results}

Sixty-two patients were enrolled in the study. Demographic information is presented in Table 1.

Forty-four (71\%) patients had pneumonia based on $\mathrm{CT}$. Ultrasound was positive in 40 of the 44 patients 
Table 1 Patient demographics

\begin{tabular}{lll}
\hline & $\begin{array}{l}\text { Pneumonia } \\
(n=44)\end{array}$ & $\begin{array}{l}\text { No pneumonia } \\
(n=18)\end{array}$ \\
\hline $\begin{array}{ll}\text { Age range, year } \\
\text { 30-39 }\end{array}$ & $3(7)$ & $2(11)$ \\
$40-49$ & $9(20)$ & $3(17)$ \\
$50-59$ & $10(23)$ & $3(17)$ \\
$60-69$ & $13(30)$ & $4(22)$ \\
$70-79$ & $6(14)$ & $2(11)$ \\
$\geq 80$ & $3(7)$ & $4(22)$ \\
Mean Age (SD) & $58.5(13.8)$ & $61.2(16.3)$ \\
Women & $26(59)$ & $7(39)$ \\
History of chronic obstructive & $18(41)$ & $11(61)$ \\
pulmonary disease & & $1(6)$ \\
History of tuberculosis & $4(9)$ & \\
\hline
\end{tabular}

Values are presented as number (\%)

with pneumonia, demonstrating a sensitivity of $91 \%$. Chest X-ray was positive in 32 of the 44 patients with pneumonia, yielding a sensitivity of $73 \%$ (Table 2 ). The sensitivity of ultrasound was significantly better than chest X-ray $(p=0.01)$. Specificity of ultrasound and chest $\mathrm{X}$-ray were similar at 61 and $50 \%$ respectively $(p=0.62)$. The positive predictive value of lung ultrasound was $85 \%$ and chest X-ray was $78 \%$. The negative predictive value of ultrasound was $73 \%$ while chest X-ray was $43 \%$. The positive likelihood ratio for diagnosing pneumonia with lung ultrasound was 2.34, while the negative likelihood ratio was 0.15 . Chest X-ray had a positive and negative likelihood ratio of 1.45 and 0.55 respectively.

In patients with a false-positive ultrasound, CT diagnoses were the following: bronchiectasis with fibrosis $(n=3)$, interstitial lung disease $(n=2)$, tuberculosis $(n=1)$, and normal $(n=1)$. In the one patient with a normal CT scan, lung ultrasound was positive based on $\mathrm{B}$ lines in the posterior zone only.

Inter-rater reliability between ultrasound interpretations by the sonographer and expert reviewer was 0.79 , demonstrating very good agreement.

Clinicians performed lung ultrasound in an average of $7 \mathrm{~min} 9 \mathrm{~s}$ (SD $1 \mathrm{~min} 57 \mathrm{~s}$ ). The time it took for patients to get a chest X-ray was $117 \mathrm{~min}$ (SD $56 \mathrm{~min}$ ). This time

Table 2 Results of chest X-ray and lung ultrasound compared with CT

\begin{tabular}{cll}
\hline & CT positive $(n=44)$ & CT negative $(n=18)$ \\
\hline Chest X-ray & 32 & 9 \\
Positive & 12 & 9 \\
Negative & & \\
Ultrasound & 40 & 7 \\
Positive & 4 & 11 \\
Negative & & \\
\hline
\end{tabular}

did not include the time required for film printing and pickup for clinician review.

\section{Discussion}

To our knowledge, this is the first study to provide evidence that the sensitivity of lung ultrasound is superior to chest X-ray for diagnosing pneumonia in a lowincome country.

Lung ultrasound demonstrated a higher sensitivity for the diagnosis of pneumonia compared to chest X-ray. Previous studies have found similar results regarding the sensitivity of ultrasound for pneumonia. In recent metaanalyses, Long et al. found a pooled sensitivity of $88 \%$ for lung ultrasound and Ye et al. found a pooled sensitivity of $95 \%$ [7, 22]. However, in these meta-analyses, the majority of studies used biased reference standards such as chest X-ray and hospital discharge diagnoses, which may confound their results. Using similar methods to our study with CT as the reference standard, Liu et al.'s study in China found similar results with the sensitivity of lung ultrasound significantly outperforming chest X-ray (94.6 versus $77.7 \%, p<0.001$ ) [8].

Ultrasound missed pneumonia in four patients. These pneumonias were also missed by chest X-ray. In all four patients, the pneumonia was located in the middle of the lung parenchyma and did not extend to the pleura. This is similar to prior studies finding that lesions not extending to the pleura are missed by ultrasound [11]. Therefore, clinicians should continue to consider close follow-up for repeat evaluation in patients with continued high suspicion for pneumonia and negative lung ultrasound.

Specificities of lung ultrasound and chest X-ray for pneumonia were similar. Lung ultrasound specificity was significantly lower than previous studies, including the two meta-analyses previously mentioned. Long et al. found a pooled specificity of $86 \%$ while Ye et. Al found a pooled specificity of $91 \%[7,22]$. Using CT as the gold standard, Liu et al. found a specificity of lung ultrasound to be significantly better than chest X-ray (99 versus $61.1 \%, p<0.001)$ for the diagnosis of pneumonia [8]. While this study was done in China, where there are also high rates of tuberculosis and chronic obstructive pulmonary disease, there were no patients with tuberculosis in the study and only $10 \%$ of patients had a diagnosis of chronic obstructive pulmonary disease, making it difficult to extrapolate these results to other areas of China and other middle- and low-income countries. The low specificity for ultrasound in our study is due to a higher prevalence of co-morbidities, including previous pulmonary tuberculosis, bronchiectasis, and chronic obstructive lung disease. Patients with history of tuberculosis often develop significant parenchymal scarring and fibrosis with bronchiectasis [23]. The CT findings 
for the majority of the false positives demonstrated significant chronic lung disease with fibrosis that made ultrasound findings consistent with consolidation [24]. Clinically, these false positives may not impact clinical care as patients with chronic lung disease may also benefit from antibiotic use in acute exacerbations [25]. However, similar to chest X-ray, low specificity may lead to over-diagnosis of pneumonia and inappropriate use of antibiotics.

One false-positive ultrasound was in a patient with active pulmonary tuberculosis confirmed by positive sputum. While tuberculosis could be considered a type of pneumonia, we included it as a false positive given the significant difference in treatment. When evaluating patients with suspected pneumonia in a setting with high rates of tuberculosis and chronic lung parenchymal disease, it is important to consider the clinical scenario to help differentiate findings on diagnostic imaging.

Our study had a high incidence of pneumonia (71\%). Other studies in developed countries evaluating ultrasound for diagnosis of pneumonia also had high rates of pneumonia, including Cortellero et al. (67.5\%) and Bourcier et al. (85\%) [9, 10]. The study in China by Liu et al. found pneumonia in $62.6 \%$ of their study population [8]. As a leading cause of death in Nepal, we believe this represents the Nepali patient population with high rates of pneumonia. Having an inexpensive, sensitive bedside test like lung ultrasound to assure clinician's do not miss this diagnosis and can more confidently rule out pneumonia with a negative test could help clinicians in this resourcelimited setting.

Lung ultrasound was able to be performed and interpreted rapidly at the bedside. In this setting, given that chest X-ray took an average of nearly $2 \mathrm{~h}$ to be performed (not including print time and physician interpretation), bedside lung ultrasound can provide a quicker diagnosis with timely and appropriate therapy. Bedside chest ultrasound is also cheaper than chest Xray, costing on average one third to half as much as chest X-ray and, therefore, providing a more sensitive test at reduced costs [26]. Additionally, its use may provide a more patient and family-centered diagnostic approach, especially in this setting where portable imaging is not available and the family is required to take the patient to radiology, which depending on severity of illness may require an oxygen tank, intravenous fluids, and wheelchair or stretcher. Increasing awareness of the diagnostic accuracy of ultrasound for pneumonia and training clinicians could improve evaluation of patients with pneumonia throughout Nepal and other resourcelimited settings.

This study has several limitations. The study was designed to evaluate a difference in sensitivity between lung ultrasound and chest X-ray, which required a sample size of 62 patients. This small sample size gives limited information regarding specificity and other diagnostic characteristics of lung ultrasound in this setting. A repeat study with a larger, more heterogeneous sample is needed to further evaluate these diagnostic characteristics, recognizing that the specificity may not perform as well in low-income countries. Also, the study was completed in one hospital in an urban city in Nepal, making it difficult to extrapolate results to other rural and resource-limited areas. Despite this, our patient population represents the general population of Nepal with its prevalence of disease. Therefore, we believe lung ultrasound would perform similarly in other settings within Nepal and settings similar to Nepal [16-18, 27]. Additionally, ultrasonography is an operator-dependent examination that requires training. The results of this paper are based on four emergency physicians trained in lung ultrasound. While the study demonstrated reliability of the ultrasound readings and subsequent results, broad implementation of lung ultrasound requires training and assessment of clinicians. There is no consensus on what level of training is required to achieve competency in lung ultrasonography for pneumonia, and the results in other settings may be different, due to different skills and experience. Despite this, these results demonstrate the benefit of including lung ultrasound in training and in implementation of guidelines for pneumonia diagnosis and treatment in Nepal and other resource-limited countries.

\section{Conclusions}

Bedside lung ultrasound demonstrated better sensitivity than chest X-ray for the diagnosis of pneumonia in Nepal. Given this diagnostic performance, lung ultrasound could be another imaging modality used to diagnose pneumonia in a resource-limited setting.

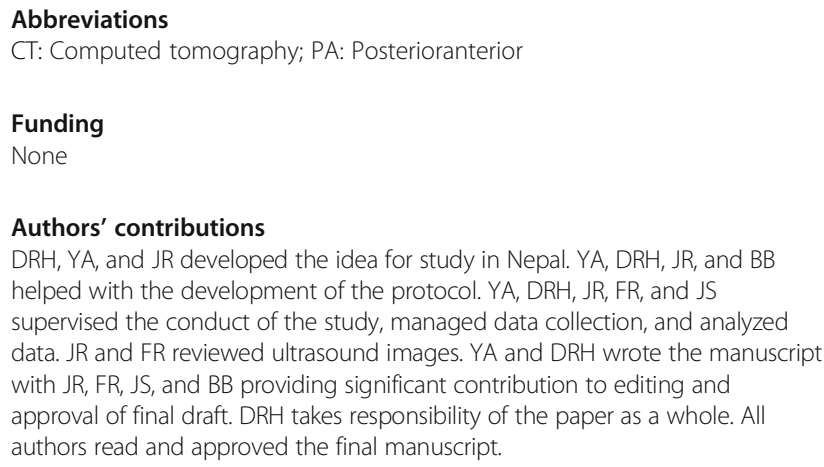

Ethics approval and consent to participate

This study was approved by both Vanderbilt University Institutional Review Board (IRB\# 161290) and Nepal Health Research Council Ethical Review Board (Reference no. 570). Written informed consent in Nepali was obtained from patients prior to enrollment. 


\section{Competing interests}

The authors declare that they have no competing interests.

\section{Publisher's Note}

Springer Nature remains neutral with regard to jurisdictional claims in published maps and institutional affiliations.

\section{Author details}

'Department of General Practice and Emergency Medicine, Patan Academy of Health Sciences, PO Box 26500, Kathmandu, Nepal. ${ }^{2}$ Department of Emergency Medicine, Vanderbilt University Medical Center, Nashville, TN, USA. ${ }^{3}$ Department of Emergency Medicine, Indiana University School of Medicine, Indianapolis, IN, USA.

Received: 20 October 2017 Accepted: 8 February 2018

Published online: 12 March 2018

\section{References}

1. World Health Organization. The 10 leading causes of death by country income group 2017 [updated 2017. Available from: http://www.who.int/ mediacentre/factsheets/fs310/en/index1.html]. Accessed 26 Apr 2017.

2. Mortensen EM, Restrepo MI, Anzueto A, Pugh JA. Antibiotic therapy and 48-hour mortality for patients with pneumonia. Am J Med. 2006; 119(10):859-64.

3. Brenner DJ, Hall EJ. Computed tomography —an increasing source of radiation exposure. N Engl J Med. 2007;357(22):2277-84.

4. Syrjala H, Broas M, Suramo I, Ojala A, Lahde S. High-resolution computed tomography for the diagnosis of community-acquired pneumonia. Clin Infect Dis. 1998;27(2):358-63.

5. Hagaman JT, Rouan GW, Shipley RT, Panos RJ. Admission chest radiograph lacks sensitivity in the diagnosis of community-acquired pneumonia. Am J Med Sci. 2009;337(4):236-40.

6. Self WH, Courtney DM, McNaughton CD, Wunderink RG, Kline JA. High discordance of chest $\mathrm{x}$-ray and computed tomography for detection of pulmonary opacities in ED patients: implications for diagnosing pneumonia. Am J Emerg Med. 2013;31(2):401-5.

7. Ye X, Xiao H, Chen B, Zhang S. Accuracy of lung ultrasonography versus chest radiography for the diagnosis of adult community-acquired pneumonia: review of the literature and meta-analysis. PLoS One. 2015; 10(6):e0130066.

8. Liu XL, Lian R, Tao YK, Gu CD, Zhang GQ. Lung ultrasonography: an effective way to diagnose community-acquired pneumonia. Emerg Med J. 2015;32(6):433-8.

9. Bourcier JE, Paquet J, Seinger M, Gallard E, Redonnet JP, Cheddadi F, et al. Performance comparison of lung ultrasound and chest $x$-ray for the diagnosis of pneumonia in the ED. Am J Emerg Med. 2014;32(2):115-8.

10. Cortellaro F, Colombo S, Coen D, Duca PG. Lung ultrasound is an accurate diagnostic tool for the diagnosis of pneumonia in the emergency department. Emerg Med J. 2012;29(1):19-23.

11. Reissig A, Copetti R, Mathis G, Mempel C, Schuler A, Zechner P, et al. Lung ultrasound in the diagnosis and follow-up of community-acquired pneumonia: a prospective, multicenter, diagnostic accuracy study. Chest. 2012:142(4):965-72

12. Pereda MA, Chavez MA, Hooper-Miele CC, Gilman RH, Steinhoff MC, Ellington $L E$, et al. Lung ultrasound for the diagnosis of pneumonia in children: a meta-analysis. Pediatrics. 2015;135(4):714-22.

13. Xia Y, Ying Y, Wang S, Li W, Shen $\mathrm{H}$. Effectiveness of lung ultrasonography for diagnosis of pneumonia in adults: a systematic review and metaanalysis. J Thorac Dis. 2016:8(10):2822-31.

14. Sippel S, Muruganandan K, Levine A, Shah S. Review article: use of ultrasound in the developing world. Int J Emerg Med. 2011:4:72

15. Wipf JE, Lipsky BA, Hirschmann JV, Boyko EJ, Takasugi J, Peugeot RL, et al. Diagnosing pneumonia by physical examination: relevant or relic? Arch Intern Med. 1999:159(10):1082-7.

16. Bhandari GP, Angdembe MR, Dhimal M, Neupane S, Bhusal C. State of noncommunicable diseases in Nepal. BMC Public Health. 2014;14:23.

17. Dye C. Global epidemiology of tuberculosis. Lancet. 2006;367(9514):938-40.

18. Gautam R, Neupane D, Karki A, Kallestrup P. Community-based management of COPD in Nepal. Lancet Respir Med. 2017;5(1):e6.
19. Grossman RF, Hsueh PR, Gillespie SH, Blasi F. Community-acquired pneumonia and tuberculosis: differential diagnosis and the use of fluoroquinolones. Int J Infect Dis. 2014;18:14-21.

20. Nyamande K, Lalloo UG, John M. TB presenting as community-acquired pneumonia in a setting of high TB incidence and high HIV prevalence. Int J Tuberc Lung Dis. 2007;11(12):1308-13.

21. Lichtenstein DA, Lascols N, Meziere G, Gepner A. Ultrasound diagnosis of alveolar consolidation in the critically ill. Intensive Care Med. 2004; 30(2):276-81.

22. Long L, Zhao HT, Zhang ZY, Wang GY, Zhao HL. Lung ultrasound for the diagnosis of pneumonia in adults: a meta-analysis. Medicine (Baltimore). 2017;96(3):e5713.

23. Kim HY, Song KS, Goo JM, Lee JS, Lee KS, Lim TH. Thoracic sequelae and complications of tuberculosis. Radiographics. 2001;21(4):839-58. discussion 59-60

24. Volpicelli G, Elbarbary M, Blaivas M, Lichtenstein DA, Mathis G, Kirkpatrick AW, et al. International evidence-based recommendations for point-of-care lung ultrasound. Intensive Care Med. 2012;38(4):577-91.

25. Vollenweider DJ, Jarrett H, Steurer-Stey CA, Garcia-Aymerich J, Puhan MA. Antibiotics for exacerbations of chronic obstructive pulmonary disease. Cochrane Database Syst Rev. 2012;12:CD010257

26. Jones BP, Tay ET, Elikashvili I, Sanders JE, Paul AZ, Nelson BP, et al. Feasibility and safety of substituting lung ultrasonography for chest radiography when diagnosing pneumonia in children: a randomized controlled trial. Chest. 2016;150(1):131-8.

27. Institute for Health Metrics and Evaluation. Global Burden of Diseases: Nepal 2010 Available from: http://www.healthdata.org/sites/default/files/files/ country_profiles/GBD/ihme_gbd_country_report_nepal.pdf. Accessed 25 July 2017

\section{Submit your manuscript to a SpringerOpen ${ }^{\circ}$ journal and benefit from:}

- Convenient online submission

- Rigorous peer review

- Open access: articles freely available online

- High visibility within the field

Retaining the copyright to your article

Submit your next manuscript at springeropen.com 